\title{
Influência da Temperatura e Radiação Ultravioleta no Desenvolvimento de Isolados de Trichoderma spp.
}

\author{
Laís Lacerda Brasil de Oliveira $^{1}$ (D), João Gutemberg Leite Moraes ${ }^{1}$, \\ Christiana de Fátima Bruce da Silva ${ }^{2}$, Alan Bernard Oliveira de Sousa ${ }^{3}$, \\ Natália Moura de Vasconcelos Beleza ${ }^{2}$, Silvio Gentil Jacinto Júnior ${ }^{1}$ \\ ${ }^{1}$ Mestrado em Climatologia e Aplicações nos Países da CPLP, Universidade Estadual do Ceará, \\ Fortaleza, CE, Brasil. \\ ${ }^{2}$ Laboratório de Patologia Pós-Colheita, Embrapa Agroindústria Tropical, Fortaleza, CE, Brasil. \\ ${ }^{3}$ Departamento de Engenharia Agrícola, Universidade Federal do Ceará, Fortaleza, CE, Brasil.
}

Recebido em: 30 de Abril de 2019 - Aceito em: 27 de Junho de 2019

\begin{abstract}
Resumo
As mudanças climáticas são uma ameaça para o setor agrícola. O objetivo do estudo foi avaliar a influência da temperatura e radiação ultravioleta (UV) no desenvolvimento in vitro de isolados de Trichoderma spp. Os experimentos foram conduzidos no Laboratório de Patologia Pós-Colheita, da Embrapa Agroindústria Tropical (Fortaleza - CE). Avaliou-se a influência da temperatura de incubação $\left(30,35\right.$ e $\left.40^{\circ} \mathrm{C}\right)$ no crescimento micelial, bem como na esporulação (conídios. $\mathrm{mL}^{-1}$ ) e na germinação (\%) dos conídios dos isolados de Trichoderma spp. (T. asperellum - SF 04, Quality WG ${ }^{\circledR} ; T$. harzianum - IBLF 006, Ecotrich WP ${ }^{\circledR}$; e T. harzianum ESALQ - 1306, Trichodermil 1306). Para a avaliação da influência da UV, as placas de Petri contendo os isolados fúngicos foram expostas à luz em diferentes intervalos de tempo $(0,2$, 4, 6 e 8 minutos) e obteve-se a germinação relativa. O isolado mais sensível à temperatura foi T. asperellum (SF 04), no qual, a temperatura de $35^{\circ} \mathrm{C}$ apresentou menor área de crescimento micelial e menor germinação relativa $(38,4 \%)$. $\mathrm{O}$ isolado T. harzianum (ESALQ 1306) apresentou a menor porcentagem de conídios germinados a $30{ }^{\circ} \mathrm{C}(63 \%)$, porém, teve o maior número a $35^{\circ} \mathrm{C}(100 \%)$. A radiação UV diminuiu a germinação dos conídios e os isolados que apresentaram maior germinação relativa ao final do período de exposição foi T. asperellum (SF 04) e T. harzianum (ESALQ 1306). Estes resultados podem demonstrar uma maior capacidade de adaptação do isolado T. harzianum (ESALQ 1306) às condições extremas de temperatura.
\end{abstract}

Palavras-chave: mudanças climáticas, sistema de produção, promoção de crescimento, controle biológico, adaptação.

\section{Influence of Temperature and UV radiation in the development of strains of}

\section{Trichoderma spp.}

\begin{abstract}
Climate change is a threat to the agricultural sector. The objective of this study was to evaluate the influence of temperature and ultraviolet radiation (UV) on the in vitro development of Trichoderma spp. The experiments were conducted at the Postharvest Pathology Laboratory, at Embrapa Agroindústria Tropical (Fortaleza - CE). It was evaluated the influence of the incubation temperature $\left(30,35\right.$ and $40{ }^{\circ} \mathrm{C}$ ) on mycelial growth, as well as sporulation (conidia.mL-1) and germination (\%) of the conidia of the Trichoderma spp. (T. asperellum - SF 04, Quality WG®; T. harzianum - IBLF 006, Ecotrich WP®; and T. harzianum ESALQ - 1306, Trichodermil 1306). To evaluate the influence of UV, the Petri dishes of the fungal strains were exposed to light at different time intervals $(0,2,4,6$ and 8 minutes) and obtained relative germination. The most temperature sensitive strain was $T$. asperellum (SF 04), in which the temperature of $35^{\circ} \mathrm{C}$ showed a lower area of mycelial growth and lower relative germination $(38.4 \%)$. The $T$. harzianum strain (ESALQ 1306) presented the lowest percentage of conidia germinated at $30{ }^{\circ} \mathrm{C}(63 \%)$, but had the highest number at $35{ }^{\circ} \mathrm{C}$ $(100 \%)$. The UV radiation decreased the germination of the conidia and the strains that had the highest germination relative to the end of the exposure period were T. asperellum (SF 04) and T. harzianum (ESALQ 1306). These results may demonstrate a higher adaptability of the T. harzianum strain (ESALQ 1306) to extreme temperature conditions.
\end{abstract}

Keywords: climate change, production system, growth promotion, biological control, adaptation.

Autor de correspondência: Laís Lacerda Brasil de Oliveira, laisbrasil@ufc.br 


\section{Introdução}

$\mathrm{O}$ aumento nas concentrações de dióxido de carbono $\left(\mathrm{CO}_{2}\right)$, metano $\left(\mathrm{CH}_{4}\right)$, monóxido de carbono $(\mathrm{CO})$, óxido nitroso $\left(\mathrm{N}_{2} \mathrm{O}\right)$, óxido de nitrogênio $\left(\mathrm{NO}_{2}\right)$ e ozônio $\left(\mathrm{O}_{3}\right)$, gases relacionados ao efeito estufa, estão contribuindo para o fenômeno das mudanças climáticas (Ghini et al., 2008). Este efeito tem recebido grande destaque da mídia e são objeto de estudo de considerável número de pesquisadores. Tem-se constatado que, não há previsão da redução da concentração de nenhum desses gases em curto prazo, antevendo dessa forma que os habitantes do planeta Terra sofrerão as consequências dessas mudanças por muitos anos (IPCC, 2013).

O Nordeste do Brasil (NEB) tem considerável vulnerabilidade às mudanças climáticas e está sujeito a alterações na distribuição de eventos extremos, veranicos, distribuição espacial de chuvas, etc., com diversos sistemas contribuindo para a variabilidade climática e possivelmente a alterações climáticas naturais e antrópicas (Kayano e Andreoli, 2009; PBMC, 2012). O estado do Ceará (CE) situa-se no NEB e apresenta clima predominantemente semi-árido. As precipitações são variáveis e no litoral de Fortaleza pode chegar a mais de 1.400/ ano (Coutinho et al., 2017). As projeções do PBMC (Painel Brasileiro de Mudanças Climáticas) para o NEB são de decréscimo entre $10 \%$ e $20 \%$ da precipitação e aumento de 0,5 a $1{ }^{\circ} \mathrm{C}$ da temperatura do ar durante as próximas três décadas (até 2040), com aumento gradual de temperatura de 1,5 a $2,5^{\circ} \mathrm{C}$ e diminuição entre $25 \%$ e $35 \%$ nos padrões de chuva no período de 2041-2070. Essas mudanças podem desencadear o processo de desertificação da caatinga e severas consequências (PBMC, 2012).

As alterações climáticas tem influência também, diretamente na produção agrícola modificando a fisiologia, o crescimento e o rendimento das culturas (Ainsworth et al., 2002; Percy et al., 2003). Além disso, podem influenciar nas relações entre patógeno/planta, ocasionando maiores problemas nas intensidades das doenças (Mcelrone et al., 2010). Em decorrência dessas mudanças, será necessário, rever os tratos culturais realizados nos cultivos, que poderão perder a eficiência e tornar a agricultura ainda mais dependente de defensivos para suprir as adversidades, resultando em maior custo na produção e, consequentemente, em toda a cadeia consumidora (Hannukkala et al., 2007).

Uma das técnicas que pode ser afetada diretamente pelas mudanças climáticas é o uso de agentes de controle biológico (antagonistas) e de promotores de crescimento de plantas. Uma vez alterado o ambiente, os reflexos serão sentidos nas populações de patógenos, nos hospedeiros, bem como nos antagonistas e nos promotores de crescimento. Estas alterações podem provocar perdas de função ou de adaptabilidade destes microrganismos benéficos (Lucon, 2008). Dentre os agentes de controle biológico e promotores de crescimento de plantas, destacam-se os fungos do gênero Trichoderma, que pertencem à família Hypocreaceae (Index fugorum, 2018). Trichoderma é um fungo saprófita, comum nos solos. Suas estruturas vegetativas são chamadas de micélios e as estruturas reprodutivas são denominadas de conídios. Este fungo apresenta uma importante função ecológica, pois participa na mineralização dos restos de folhas, caules e raízes mortas, ajudando a manter o equilíbrio do ambiente (Gams e Bisset, 1998).

Os mecanismos de ação das espécies de Trichoder$m a$ são variados, destacando-se a antibiose, competição, micoparasitismo, promoção de crescimento e a indução de resistência à doenças. Além disso, diferentes espécies de Trichoderma produzem enzimas que podem ter aplicações industriais, como polissacaridases, proteases e lipases. $\mathrm{Na}$ natureza, tais enzimas estão envolvidas na degradação da parede celular dos fitopatógenos (Lucon, 2008). Adicionalmente, esses fungos têm grande facilidade em colonizar os diversos substratos, inclusive raízes de plantas (Machado et al., 2012).

Apesar de ser um gênero encontrado em vários ambientes, alguns fatores climáticos podem ser limitantes ao fungo. Por exemplo, a temperatura ótima de crescimento da maioria dos isolados de Trichoderma está em torno de 25 a $30^{\circ} \mathrm{C}$ (Bomfim et al., 2010). Além da temperatura, a radiação ultravioleta (UV) é outro fator limitante no seu crescimento, podendo ser letal, caso estejam diretamente expostos. Dessa forma é importante escolher o melhor agente biológico que se adapte às condições abióticas (temperatura e radiação UV) as quais são expostos, para que assim tenham melhor eficiência no biocontrole (Braga et al., 2001).

Cabe salientar que, um aspecto fundamental na exploração econômica da diversidade microbiana para desenvolvimento de biodefensivos reside na descoberta de isolados com maior atividade ou mais adaptadas às condições ambientais onde esses produtos serão utilizados Os experimentos in vitro, por exemplo, permitem identificar os isolados promissores no antagonismo a patógenos, bem como na promoção do crescimento de plantas (ValadaresInglis et al., 2018). Adicionalmente, vale destacar também que, o uso de diferentes isolados de Trichoderma tem levado a aumentos significativos na porcentagem e precocidade de germinação, aumento no crescimento e produtividade de várias culturas agrícolas. Como exemplo, podem-se citar cultivos de milho (Zea mays), feijão (Phaseolus vulgaris), ervilha (Pisum sativum), entre outros (Resende et al., 2004; Hoyos-Carvajal et al., 2009).

Tendo em vista as mudanças climáticas e seus efeitos sobre a radiação UV e temperatura, é importante avaliar a influência desses fatores abióticos, in vitro, no desenvolvimento de isolados de Trichoderma, como forma de auxiliar nas metodologias propostas para testes em condições controladas. Portanto, o objetivo deste trabalho 
é avaliar in vitro a influência da temperatura e radiação UV no desenvolvimento de três isolados de Trichoderma spp.

\section{Materiais e Métodos}

\subsection{Obtenção e multiplicação do inóculo dos isolados de Trichoderma}

Os experimentos foram conduzidos no Laboratório de Patologia Pós-Colheita, localizado na Embrapa Agroindústria Tropical (Fortaleza, CE). Os isolados fúngicos para todos os ensaios foram selecionados de acordo com outros trabalhos conduzidos em diferentes regiões do país, que utilizam produtos comercias à base do ingrediente ativo de Trichoderma (Lima et al., 2016; França et al., 2017; Silva et al., 2017). Dessa forma, para a obtenção dos isolados foram utilizados 3 produtos comerciais: T. asperellum - SF 04 (Quality WG ${ }^{\circledR}$; Laboratório de BioControle Farroupilha S.A, Patos de Minas, MG, Brasil), T. harzianum - IBLF 006 (Ecotrich WP ${ }^{\circledR}$; Ballagro Agro Tecnologia Ltda., Atibaia, SP, Brasil) e T. harzianum ESALQ 1306 (Trichodermil $1306^{\circledR}$; Koppert do Brasil Sistemas Biológicos Ltda).

Para a obtenção do inóculo inicial, os produtos foram hidratados em solução salina $0,85 \%$, por cinco minutos e $1 \mathrm{~mL}$ de cada suspensão foram depositados individualmente no centro de placas de Petri, contendo meio de cultura BDA (batata dextrose ágar). Após o procedimento, as placas foram incubadas na temperatura de $28^{\circ} \mathrm{C}$ e fotoperíodo de $12 \mathrm{~h}$ claro/ $12 \mathrm{~h}$ escuro.

\subsection{Influência de diferentes temperaturas no crescimento micelial de isolados de Trichoderma}

Para este ensaio, foram inoculadas placas com meio BDA contendo um disco de micélio de $6 \mathrm{~mm}$, com 7 dias de crescimento de cada isolado de Trichoderma (T. asperellum - SF 04; T. harzianum - IBLF 006 e T. harzianum ESALQ 1306), disposto no centro da placa. As placas foram submetidas às temperaturas de 30,35 e $40^{\circ} \mathrm{C}$ em incubadoras do tipo BOD. Foram incubadas 20 placas de Petri de cada isolado distribuídos igualmente nas 4 prateleiras, totalizando 60 placas em cada BOD, nas respectivas temperaturas.

O crescimento micelial foi mensurado diariamente, com auxílio de paquímetro e foi obtido o diâmetro médio de crescimento micelial, em dois eixos ortogonais (média das duas medidas diametralmente opostas), até que nas placas incubadas na temperatura de $30{ }^{\circ} \mathrm{C}$ (temperatura ideal de crescimento), o crescimento micelial de uma das colônias do fungo atingisse a borda da placa. A partir daí calculou-se a área de crescimento micelial, em $\mathrm{mm}^{2}$.

Posteriormente, obteve-se a Área Abaixo da Curva de Crescimento Micelial, expressa $\mathrm{em}^{2}$, adaptado de Campbell e Madden (1990):

$$
\mathrm{AACM}=\sum_{i}^{n-1} \frac{(y i+y i+1)}{2}(\mathrm{t} i+1-\mathrm{t} i)
$$

em que y $i+\mathrm{y} i+1$ são os valores de crescimento da colônia observados em duas avaliações consecutivas; ti o intervalo entre as avaliações e $n$ a duração do período de avaliação.

\subsection{Influência de diferentes temperaturas na capacidade de esporulação de isolados de Trichoderma}

Para obter os dados de esporulação, foram selecionadas 4 placas representativas de cada isolado, nas condições de melhor crescimento nas temperaturas de 30 e $35^{\circ} \mathrm{C}$, com 8 dias de crescimento em meio BDA. Foram obtidas suspensões de conídios de maneira asséptica, a partir da lavagem das placas com $20 \mathrm{~mL}$ de solução salina estéril, a $0,85 \%$. A filtração foi realizada com auxílio de gaze e, a suspensão foi depositada em béqueres estéreis. Então, procedeu-se a contagem dos conídios de Trichoderma, com auxílio de Câmara de Neubauer, no microscópio de luz (40x) e a concentração foi expressa em conídios $/ \mathrm{mL}$.

\subsection{Influência de diferentes temperaturas na germinação dos conídios de isolados de Trichoderma}

$\mathrm{O}$ teste de germinação foi realizado assepticamente obtendo-se uma suspensão de conídios, à partir da lavagem de cada placa com $20 \mathrm{~mL}$ de solução salina estéril, a $0,85 \mathrm{~g} / \mathrm{L}$ e filtragem com gaze. A contagem foi conduzida em microscópio de luz (40x), com auxílio da Câmara de Neubauer. Para tal foi necessário ajustar a concentração dos conídios, para $1 \times 10^{5}$ conídio $\mathrm{mL}^{-1}$, através de diluições seriadas.

Após a obtenção da suspensão de conídios e diluição, foram dispostos $100 \mu \mathrm{l}$ no centro de cada uma das placas de Petri, contendo meio Ágar-Água 2\%, espalhando-se com alça de Drigalski até secagem. Então, procedeu-se a incubação das placas nas temperaturas de 30,35 e $40{ }^{\circ} \mathrm{C}$, em BOD por $24 \mathrm{~h}$, com fotoperíodo de $12 \mathrm{~h}$ claro/ $12 \mathrm{~h}$ escuro. Após decorrido esse tempo, cortou-se um fragmento de aproximadamente $1 \times 1 \mathrm{~cm}$ do meio de cultura, com auxílio de bisturi estéril para dispensar na lâmina e adicionou-se uma gota de corante Azul de Aman, que contém lactofenol, capaz de paralisar a germinação.

As lâminas foram avaliadas no microscópio de luz (40x), em quadruplicata e a cada 100 conídios contabilizados, aleatoriamente, foi registrado o número daqueles que haviam germinado, ou seja, aqueles em que o tubo germinativo era igual ou maior do que a largura do conídio. Os dados foram expressos em germinação relativa (\%).

\subsection{Influência de diferentes intervalos de exposição à radiação UV na germinação dos conídios de isolados de Trichoderma}

$\mathrm{Na}$ avaliação do efeito da luz ultravioleta na germinação de conídios dos isolados de Trichoderma, foram 
realizados testes de exposição das placas de Petri, contendo o crescimento dos isolados. Primeiramente, obteve-se uma suspensão de conídios a partir da lavagem das placas com $20 \mathrm{~mL}$ de solução salina estéril, a $0,85 \mathrm{~g} / \mathrm{L}$ e filtragem com gaze. Após ajuste da concentração para $1 \times 10^{5}$ conídios $\mathrm{mL}^{-1}$, através de diluições seriadas, foram dispostos $100 \mu \mathrm{L}$ no centro de cada uma das placas (duplicata) contendo meio Ágar-Água 2\%, espalhando-se com alça de Drigalski até secagem.

As placas de Petri foram expostas abertas à luz UV de $217 \mathrm{~V}$ com comprimento de onda entre 230 e $270 \mathrm{~nm}$ do fluxo laminar Pachane ${ }^{\circledR}$, modelo PA 310 ECO, nos intervalos de $0,2,4,6$ e 8 minutos. Os testes preliminares demonstraram que no tempo de 10 minutos, não houve germinação dos conídios. Ao final de cada intervalo, as placas eram envoltas em papel alumínio e mantidas na câmara de fluxo laminar durante toda a exposição.

Então, procedeu-se a incubação das placas na temperatura de $30{ }^{\circ} \mathrm{C}$, em BOD por $24 \mathrm{~h}$, com fotoperíodo de $12 \mathrm{~h}$ claro/12 h escuro. Após decorrido esse tempo, cortou-se um fragmento de aproximadamente $1 \times 1 \mathrm{~cm}$ do meio de cultura, com auxílio de bisturi estéril. $\mathrm{O}$ fragmento foi dispensado em lâmina e adicionou-se uma gota de corante Azul de Aman, para paralisar a germinação. As lâminas foram analisadas no microscópio de luz (40x), em quadruplicata e a cada 100 conídios contabilizados, aleatoriamente, foi registrado o número daqueles que haviam germinado. Os dados foram expressos em germinação relativa $(\%)$.

\subsection{Análise estatística}

Para o ensaio de crescimento micelial, o delineamento experimental foi em blocos casualizados. Cada bloco correspondeu a uma prateleira da BOD, onde ficavam acondicionados 5 placas de cada um dos três isolados estudados. Dessa forma, foram utilizadas 20 placas para cada isolado, em cada BOD, nas temperaturas de 30, 35 e $40{ }^{\circ} \mathrm{C}$. Os dados de crescimento micelial, esporulação e germinação obtidos foram submetidos à análise de

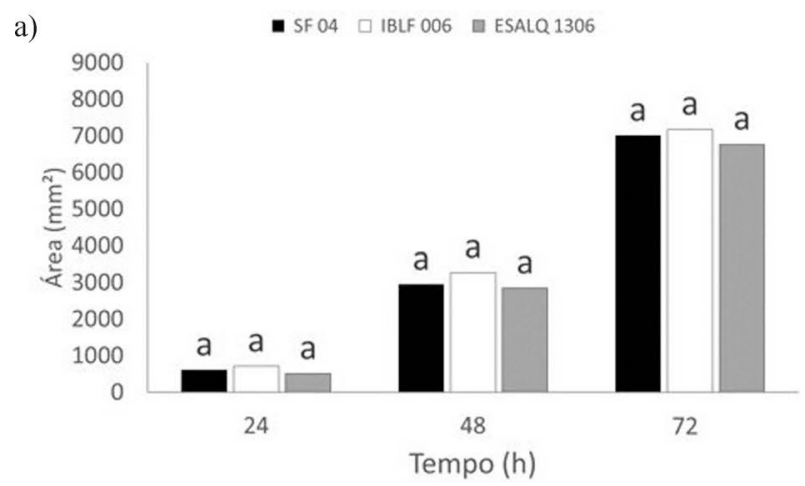

variância e as médias comparadas pelo teste de Tukey, a $5 \%$ de probabilidade, utilizando o programa estatístico Sisvar $^{\circledR}$.

Para o teste de germinação na luz UV foram calculados os desvios padrão.

\section{Resultados e Discussão}

\subsection{Influência de diferentes temperaturas no crescimento micelial de isolados de Trichoderma}

No que se refere ao crescimento micelial na temperatura de $30^{\circ} \mathrm{C}$ (Fig. 1a), não houve diferença significativa no crescimento micelial entre os isolados, durante os dias de avaliação. Porém, na temperatura de $35{ }^{\circ} \mathrm{C}$ a $72 \mathrm{~h}$ de incubação (Fig. 1b) houve incrementos no crescimento de T. harzianum (ESALQ 1306) em relação aos demais, apresentando uma área de crescimento médio de $1329,48 \mathrm{~mm}^{2}$. Enquanto que, T. asperellum (SF 04) apresentou-se mais sensível ao aumento de temperatura, com uma área de crescimento médio de 714,52 $\mathrm{mm}^{2}$. Resultados semelhantes foram constatados em vários estudos. Por exemplo, Koike e Lucon (2003) observaram que nas temperaturas de $30{ }^{\circ} \mathrm{C}$, o fungo Trichoderma teve pleno crescimento micelial, alcançando as bordas da placa de Petri, após 72 horas de incubação. Estes resultados também foram observados por Mukherjee e Raghu (1997), onde foi demonstrado que o Trichoderma não foi muito eficaz na supressão de Sclerotium rolfsii a temperaturas acima de $30^{\circ} \mathrm{C}$, apresentando crescimento reduzido

Após $72 \mathrm{~h}$ de incubação, os isolados que foram submetidos a temperatura de $30^{\circ} \mathrm{C}$, apresentaram crescimento micelial ótimo e atingiram a borda da placa, ou seja, apresentaram uma área de crescimento micelial média de $6978,13 \mathrm{~mm}^{2}$. Enquanto, nas temperaturas de 35 e $40^{\circ} \mathrm{C}$, os isolados de Trichoderma apresentaram seu desenvolvimento afetado pelo aumento de temperatura. Bomfim et al. (2010) observaram que o crescimento ótimo das colônias de isolados de Trichoderma ocorreu na

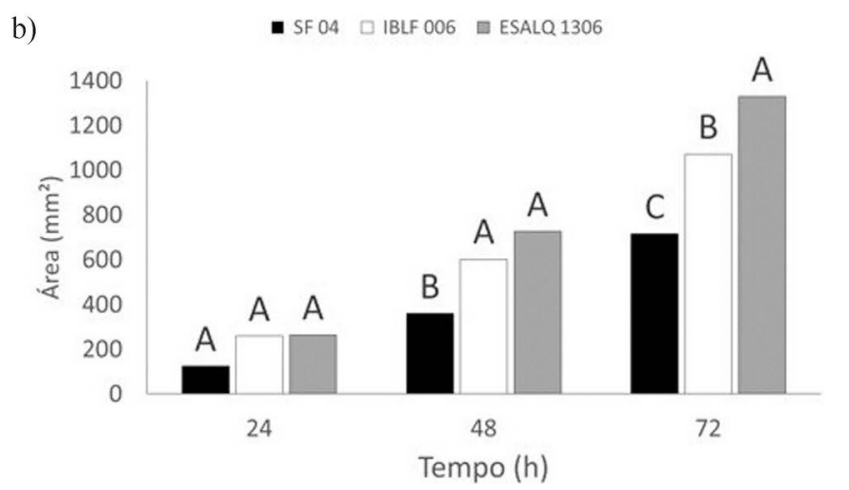

Figura 1 - Área do Crescimento micelial $\left(\mathrm{mm}^{2}\right)$ dos isolados de Trichoderma, na temperatura de $30{ }^{\circ} \mathrm{C}$ (a) e $35{ }^{\circ} \mathrm{C}$ (b), em função do tempo (horas). Isolados: SF04 (T. asperellum), IBLF006 (T. harzianum) e ESALQ1306 (T. harzianum). Médias seguidas pela mesma letra não diferem estatisticamente pelo teste Tukey a $5 \%$ de probabilidade 
temperatura de $30^{\circ} \mathrm{C}$ e, que após $48 \mathrm{~h}$ o crescimento radial atingiu a borda da placa. Nos experimentos desses autores, foi possível observar que nas temperaturas de 25 e $30{ }^{\circ} \mathrm{C}$ não houve diferenças significativas no crescimento dos isolados.

$\mathrm{O}$ isolado T. harzianum (IBLF 006) demonstrou uma área de crescimento micelial de $7168,60 \mathrm{~mm}^{2}$, que é um valor intermediário em comparação com os outros dois isolados estudados. Além disso, observou-se variações sutis no crescimento dos isolados na temperatura de $30^{\circ} \mathrm{C}$ e variações bruscas em relação ao aumento de temperatura. $\mathrm{Na}$ temperatura de $35^{\circ} \mathrm{C}$ todos os parâmetros avaliados apresentaram menores resultados do que a $30^{\circ} \mathrm{C}$.

Quanto a área abaixo da curva de crescimento micelial (AACCM), que considera o crescimento da colônia em $\mathrm{cm}^{2}$ em relação ao dia anterior, na temperatura de $30^{\circ} \mathrm{C}$ observou-se uma média de crescimento de $71,06 \mathrm{~cm}^{2}$, bem superior a média constatada na na temperatura de $35^{\circ} \mathrm{C}$, que foi de 12,97 $\mathrm{cm}^{2}$. Entretanto, é possível observar que todos os valores foram bastante semelhantes, dentro de cada temperatura, sem diferença significativa (Fig. 2).

Estas diferenças de crescimento nas diferentes temperaturas, podem ocorrer devido as características de crescimento das espécies de Trichoderma, que possuem crescimento rápido, geralmente em poucas horas ocupando toda a superfície da placa de Petri (Carabi-Adell, 2003). Em outro estudo, Jackisch-Matsuura e Menezes (1999) observaram taxas de crescimento micelial semelhantes entre os isolados de $T$. viride e $T$. harzianum, quando os mesmos foram antagonistas a Pythium aphanidermatum.

No referido estudo, não houve nenhum crescimento micelial dos isolados, na temperatura de $40{ }^{\circ} \mathrm{C}$ (Fig. 3). Isso pode estar relacionado, por exemplo, a outros fatores que podem interferir no crescimento/desenvolvimento do fungo. Por exemplo, em condições de campo, com presença de umidade, o fungo tem pleno desenvolvimento nas temperaturas que chegam até a ultrapassar os $40{ }^{\circ} \mathrm{C}$, no

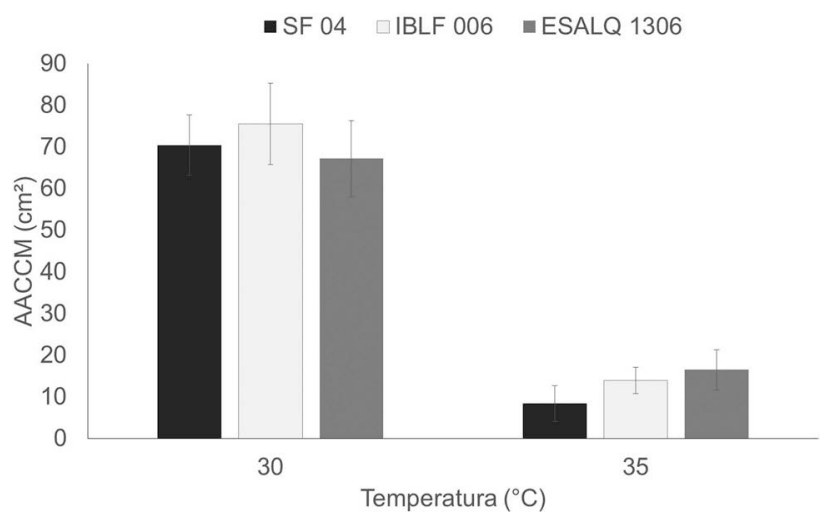

Figura 2 - Área Abaixo da Curva do Crescimento Micelial (AACCM) $\left(\mathrm{cm}^{2}\right)$ dos isolados de Trichoderma. Isolados: SF04 (T. asperellum), IBLF006 (T. harzianum) e ESALQ1306 (T. harzianum).
Nordeste brasileiro (Alves e Campos, 2003). Em outro trabalho, Guimarães et al. (2016) também constataram em estudos in vitro a ausência de crescimento de isolados de Trichoderma, diferentes dos utilizados neste estudo, na temperatura de $40{ }^{\circ} \mathrm{C}$. Para Dennis e Webster (1971) os isolados de uma mesma espécie, embora sejam morfologicamente semelhantes, podem diferir fisiologicamente e atuarem de maneira diferente quanto ao antagonismo, dependendo, portanto, mais das características fisiológicas do isolado que da espécie propriamente dita. Além da possível variabilidade genética inerente a cada isolado, existem também fatores externos como equilíbrio nutricional e influência de temperatura que afetam o desenvolvimento.

No referido estudo, as diferenças de temperatura proporcionaram as variações observadas na AACCM dos isolados de Trichoderma spp.

\subsection{Influência de diferentes temperaturas na capacidade de esporulação dos conídios de isolados de Trichoderma}

No preparo das suspensões de conídios, ao final de 8 dias, foi possível observar que a esporulação dos isolados incubados na temperatura de $30^{\circ} \mathrm{C}$ apresentou uma média de $2,27 \times 10^{7}$ conídios. $\mathrm{mL}^{-1}$ e o isolado T. asperellum (SF 04) apresentou valores significativamente menores $(1,84 \mathrm{x}$ $10^{7}$ conídios.mL $L^{-1}$ ) (Fig. 4a). Na temperatura de $35{ }^{\circ} \mathrm{C}$ a

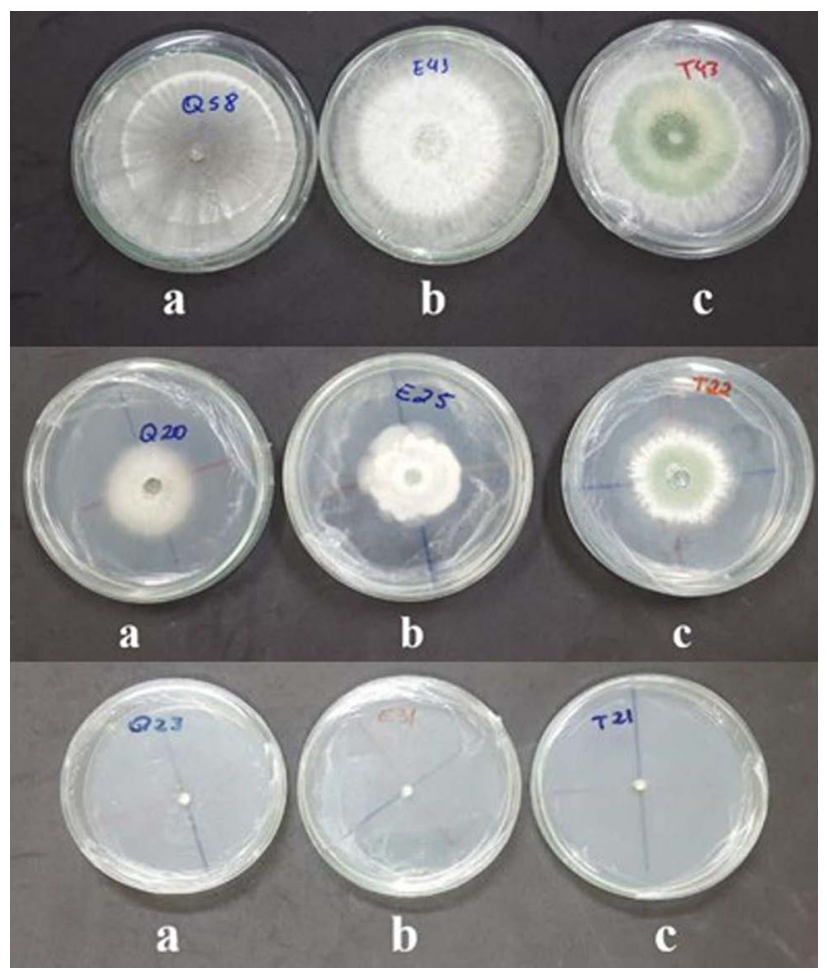

Figura 3 - Placas dos isolados de Trichoderma, com $72 \mathrm{~h}$ de incubação nas temperaturas de 30,35 e $40{ }^{\circ} \mathrm{C}$, respectivamente. Isolados: (a) SF04 (T. asperellum), (b) IBLF006 (T. harzianum) e (c) ESALQ1306 (T. harzianum). Fotos: Laís Brasil. 
esporulação foi de $3,6 \times 10^{5}$ conídios. $\mathrm{mL}^{-1}$ em média e e o isolado T. asperellum (SF 04) apresentou valores significativamente maiores $\left(5,2 \times 10^{5}\right.$ conídios. $\left.\mathrm{mL}^{-1}\right)$ (Fig. 4b).

Vale ressaltar que, como não houve crescimento micelial a $40{ }^{\circ} \mathrm{C}$, também não houve esporulação de nenhum dos isolados testados. Koike e Lucon (2003) observaram valores de $2,3 \times 10^{6}$ conídios. $\mathrm{mL}^{-1}$ de esporulação de Trichoderma, quando cultivado em meio de cultura, contendo como componente o trigo, na temperatura de $27^{\circ} \mathrm{C}$. Estes valores são semelhantes, aos constatados no referido estudo, utilizando como meio de cultura o BDA (batata dextrose ágar).

\subsection{Influência de diferentes temperaturas na germinação dos conídios de isolados de Trichoderma}

$\mathrm{O}$ teste de germinação demonstrou que o isolado $T$. harzianum (ESALQ 1306) apresentou a maior porcentagem de conídios germinados na temperatura de $35^{\circ} \mathrm{C}$. Na temperatura de $30^{\circ} \mathrm{C}$ foi constatado os menores valores de conídios germinados (Fig. 5). Estes resultados podem demonstrar uma maior capacidade de adaptação do isolado T. harzianum - ESALQ 1306 às condições extremas de temperatura, visto que em campos de produção as temperaturas do solo, onde o microrganismo se estabelece podem ultrapassar $30^{\circ} \mathrm{C}$ (Patrício, 2007).

$\mathrm{Na}$ temperatura de $40{ }^{\circ} \mathrm{C}$ não houve germinação dos conídios de nenhum dos isolados testados, pois não houve crescimento micelial e posterior formação dos conídios para a realização do teste.

\subsection{Influência de diferentes intervalos de exposição à luz UV na germinação dos conídios de isolados de Trichoderma.}

A luz UV afetou negativamente a germinação dos conídios de Trichoderma spp. (Fig. 6). Nos primeiros dois minutos de exposição, o isolado T. asperellum (SF 04) apresentou a menor germinação relativa (56\%) em relação aos demais isolados. Ao final dos oito minutos de exposi-

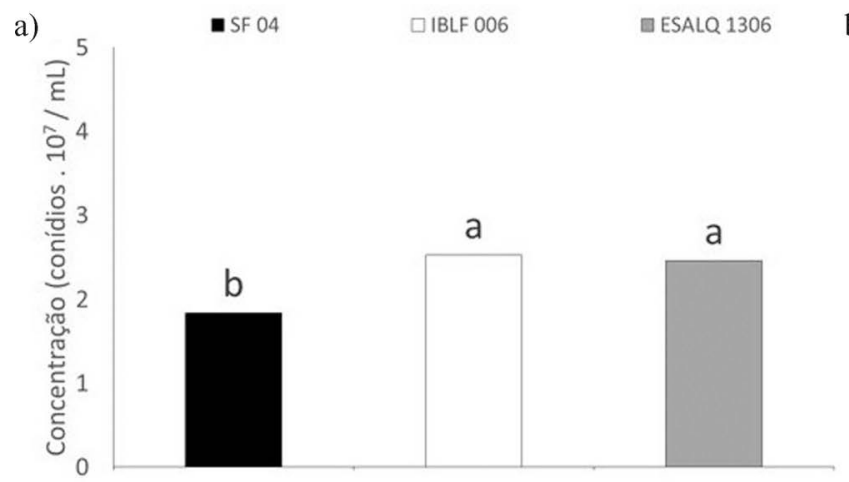

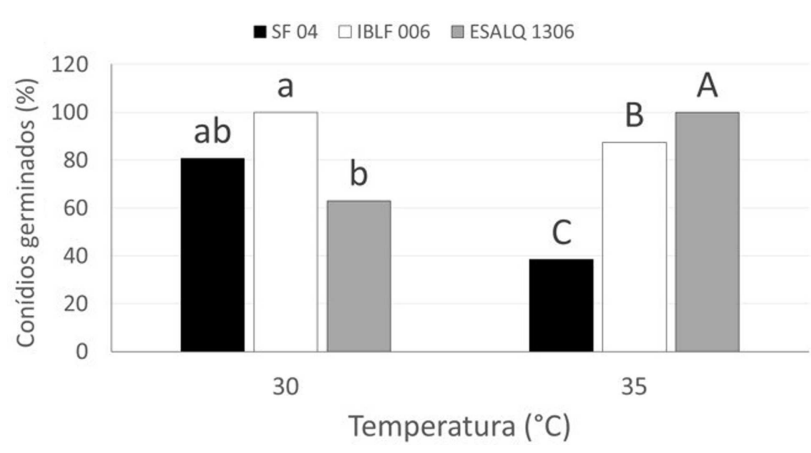

Figura 5 - Influência da temperatura na germinação dos conídios de isolados de Trichoderma. Isolados: SF04 (T. asperellum), IBLF006 (T. harzianum) e ESALQ1306 (T. harzianum). Médias seguidas pela mesma letra não diferem estatisticamente pelo teste Tukey a $5 \%$ de probabilidade. As comparações foram feitas dentro das mesmas temperaturas, por isso diferem em letras maiúsculas e minúsculas.

ção, T. harzianum (IBLF 006) teve uma queda brusca na germinação (2\%) e foi o isolado mais sensível a luz UV.

Os efeitos deletérios da radiação UV-B sobre fungos antagonistas foram também observados em Metarhizium anisopliae, Lecanicillium lecanii e Clonostachys rosea (Braga et al., 2001; Braga et al., 2002; Costa et al., 2012). Os resultados desses trabalhos assemelham-se ao do presente estudo.

Os resultados obtidos no estudo demonstram também a sensibilidade de isolados de Trichoderma ao UV-B e sugerem a necessidade de formulações com protetores anti-UV-B ou a recomendação de aplicação em períodos ou condições adequadas. Conforme Demirci et al. (2011), muitos fatores devem ser analisados para escolha de um isolado visando sua aplicação a campo. Nesse aspecto, os testes de desenvolvimento in vitro são importantes para se obter uma avaliação prévia dos isolados e saber quais fatores interferem negativamente no seu crescimento.

\section{Conclusões}

A temperatura de $35^{\circ} \mathrm{C}$ não apresentou influência no crescimento micelial, esporulação e germinação dos

b)

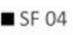

ㅁIBLF 006

๑ESALQ 1306

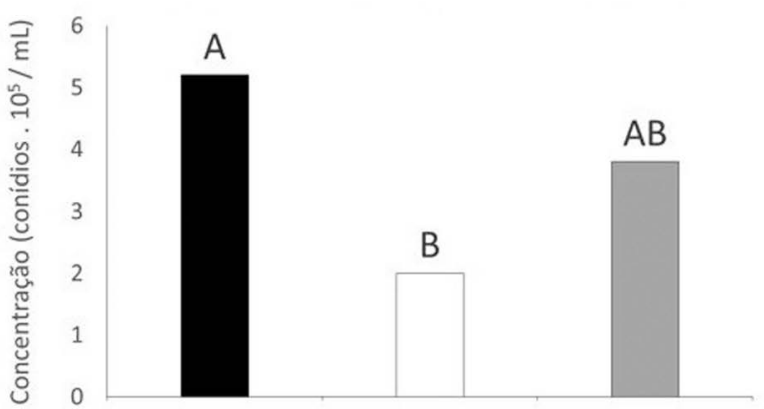

Figura 4 - Esporulação dos isolados de Trichoderma nas temperaturas de $30^{\circ} \mathrm{C}$ (a) e $35^{\circ} \mathrm{C}$ (b). Isolados: SF04 (T. asperellum), IBLF006 (T. harzianum) e ESALQ1306 (T. harzianum). Médias seguidas pela mesma letra não diferem estatisticamente pelo teste Tukey a $5 \%$ de probabilidade. 


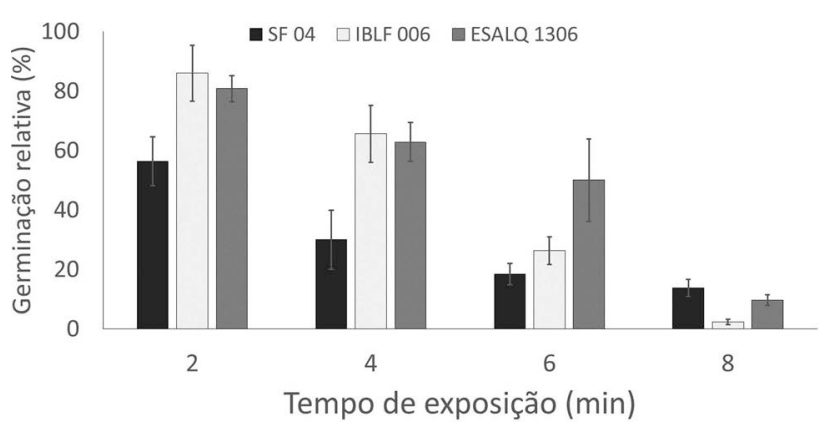

Figura 6 - Influência da radiação UV na germinação dos conídios de isolados de Trichoderma. Isolados: SF04 (T. asperellum), IBLF006 (T. harzianum) e ESALQ1306 (T. harzianum).

conídios de T. harzianum (ESALQ 1306). Os demais isolados demonstraram-se sensíveis aos incrementos de temperatura. A temperatura de $40{ }^{\circ} \mathrm{C}$ influenciou negativamente o desenvolvimento de todos os isolados.

A radiação UV diminuiu a germinação dos conídios e os isolados que apresentaram maior germinação relativa ao final do período de exposição foi T. asperellum (SF 04) e T. harzianum (ESALQ 1306). Estes resultados podem demonstrar uma maior capacidade de adaptação do isolado T. harzianum (ESALQ 1306) às condições extremas de temperatura.

\section{Referências}

AINSWORTH, E.A.; DAVEY, P.A.; BERNACCHI, C.J.; DERMODY, O.C.; HEATON, E.A.; MOORE, D.J.; MORGAN, P.B.; NAIDU, S.L.; YOO, H.S.; ZHU, R.X.G.; CURTIS, P. S.; LONG, S. P. A meta-analysis of elevated $\left[\mathrm{CO}_{2}\right]$ effects on soybean (Glycine max) physiology, growth and yield. Global Change Biology, v. 8, n. 8, p. 695-709, 2002.

ALVES, F.R.; CAMPOS, V.P. Efeitos da temperatura sobre a atividade de fungos no controle biológico de Meloidogyne javanica e $M$. incognita raça 3. Ciência e agrotecnologia, v. 27, n. 1, p. 91-97, 2003.

BRAGA, G.U.L.; FLINT, S.D.; MESSIAS, C.L.; ANDERSON, A.J.; ROBERTS, D.W. Effect of UV-B on conidia and germlings of the entomopathogenic hyphomycete Metarhizium anisopliae. Mycological Research, v. 105, n. 4, p. 874-882, 2001.

BRAGA, G.U.; RANGEL, D.E.; FLINT, S.D.; MILLER, C.D.; ANDERSON, A.J.; ROBERTS, D.W. Damage and recovery from UV-B exposure in conidia of the entomopathogens Verticillium lecani and Aphanocladium album. Mycologia, v. 94, n. 6, p. 912-920, 2002.

BOMFIM, M.P.; SÃO JOSÉ, A.R.; REBOUÇAS, T.N.H.; ALMEIDA, S.S.; SOUZA, I.V.B.; DIAS, N.O. Avaliação antagônica in vitro e in vivo de Trichoderma spp. a Rhizopus stolonifer em maracujazeiro amarelo. Summa Phytopathologica, v. 36, n. 1, p. 61-67, 2010.

CAMPBELL, C.L.; MADDEN, L. Introduction to plant disease epidemiology. New York: John Wiley \& Sons, 1990. $532 \mathrm{p}$.
CARABI-ADELL. Uma técnica simples para observação microscópica de Trichoderma spp. (Hypocreales) em cultura de lâminas. In: Reunião Atual do Instituto Biológico, 15. Arquivos do Instituto Biológico, v. 70 Suplemento, p. 3537, 2003.

COSTA, L.B.; RANGEL, D.E.N.; MORANDI, M.A.B., BETTIOL, W. Impact of UV-B radiation on Clonostachys rosea germination and growth. World Journal of Microbiology and Biotechnology, v. 28, p. 2497-2504, 2012.

COUTINHO, M.D.L.; COSTA, M.S.; GOMES, A.C.S.; MORAIS, M.D.C.; JACINTO, L.V.; LIMA, K.C.; SAKAMOTO, M. S. Estudo de Caso: Evento Extremo no Estado do Ceará entre os dias 03 e 04 de Janeiro de 2015. Revista Brasileira de Climatologia, v. 20, n. 13, p. 182-198, 2017.

DEMIRCI, E.; DANE, E.; EKEN, C. In vitro antagonistic activity of fungi isolated from sclerotia on potato tubers against Rhizoctonia solani. Turkish Journal of Biology, v. 35, p. 457-462, 2011.

DENNIS, C.; WEBSTER, J. Antagonistic properties of species groups of Trichoderma. Production of non volatile metabolites. Transactions of the British Mycological Society, v. 57, p. 25-39, 1971.

FRANÇA, D.V.C.; KUPPER, K.C.; MAGRI, M.M.R.; GOMES, T.M.; ROSSI, F. Trichoderma spp. isolates with potential of phosphate solubilization and growth promotion in cherry tomato1. Pesquisa Agropecuária Tropical, v. 47, n. 4, p. 360-368, 2017.

GAMS, W.; BISSET, J.I.T.G. Morphology and identification of Trichoderma. In: KUBICEK, C.P.; HARMAN, G.E.; ONDIK, K.L. Trichoderma and Gliocladium: Basic Biology, tTxonomy, and Genetics, p. 3-4, 1998.

GHINI, R.; HAMADA, E.; BETTIOL, W. Climate change and plant diseases. Scientia Agricola, Piracicaba, n. 65, p. 98107, 2008.

GUIMARÃES, G.R.; FERREIRA, D.S.F; GALVÃO, C.S.; MELLO, S.C.M.; CARVALHO, D.D.C. Ação de metabólitos voláteis e não voláteis de Trichoderma harzianum sobre o crescimento de Cladosporium herbarum. Pesquisa Agropecuária Pernambucana, Recife, v. 21, n. 1, p. 7-11, 2016.

HANNUKKALA, A.O.; KAUKORANTA, T.; LEHTINEN, A.; RAHKONEN, A. Late-blight epidemics on potato in Finland, 1933-2002: increased and earlier occurrence of epidemics associated with climate change and lack of rotation. Plant Pathology, Londres, v. 56, n. 1, p. 167-176, 2007.

HOYOS-CARVAJAL, L.; ORDUZ, S.; BISSETT, J. Growth stimulation in bean (Phaseolus vulgaris L.) by Trichoderma. Biological Control, Texas, v. 51, p. 409-416, 2009.

INDEX FUGORUM. Classificação filogenética de fungos. Disponível em: http://www.indexfungorum.org/names/Name sRecord.asp?RecordID=538994. Acesso em: 18 set 2018.

INTERGOVERNMENTAL PANEL ON CLIMATE CHANGE (IPCC). 2013. Climate Change 2013: The Physical Science Basis. Contribution of Working Group I to the Fifth Assessment Report of the Intergovernmental Panel on Climate Change In: STOCKER, T.F.; QUIN, D.; PLATTNER G.K.; TIGOR, M.; ALLEN, S.K.; BOSCHUNG, J.; NAUELS, A.; XIA Y.; BEX V.; MIDGLEY, P.M. (Eds.). Fifth Assessment Report: Climate Change. New York, USA: Cambridge University Press, 2013. 1535 p. 
JACKISCH-MATSUURA, A., MENEZES, M. Efeito de Trichoderma spp. no controle de Pythium aphanidermatum em fumo (Nicotiana tabacum). Summa Phytopathologica, v. 25, n. 2, p.161-164, 1999.

KAYANO, M.T.; ANDREOLI, R.V. Tempo e clima no Brasil. CAVALCANTI, I.F.A., FERREIRA, N.J., SILVA, M.G.A. J., DIAS, M.A.F.S. (org.), Cap. 14. São Paulo: Oficina de Textos, 2009.

KOIKE, C.M.; LUCON, C.M.M. Efeito de diferentes fatores na esporulação e crescimento de isolados de Trichoderma spp. Arquivos do Instituto Biológico, v. 70, suplemento 3, p. 96-99, 2003.

LIMA, M.L.P.; VAZ, M.C.A.; SILVA, A.S.; SOUZA, K.A.; TUÑON, G.I.L. In vitro confrontation of Trichoderma spp. isolates with phytopathogenic and non-phytopathogenic fungi. Revista de Agricultura Neotropical, v. 3, n. 2, p. 18, 2016.

LUCON, M.M.C. Trichoderma no controle de doenças de plantas causadas por patógenos de solo. Comunicado Técnico do Instituto Biológico, n. 77, 2008.

MACHADO, D.F.M.; PARZIANELLO, F.R.; SILVA, A.C.F.; ANTONIOLLI, Z.I. Trichoderma no Brasil: o fungo e o bioagente. Revista de Ciências Agrárias, v. 35, n. 1, p. 274-288, 2012.

MCELRONE, A.J.; HAMILTON, J.G.; KRAFNICK, A.J.; ALDEA, M.; KNEPP, R.G.; DELUCIA, E.H. Combined effects of elevated $\mathrm{CO}_{2}$ and natural climatic variation on leaf spot diseases of redbud and sweetgum trees. Environmental Pollution, v. 158, n. 1, p. 108-114, 2010.

MUKHERJEE, P.K.; RAGHU, K. Effect of temperature on antagonistic and biocontrol potencial of Trichoderma sp. on Sclerotium rolfsii. Mycopathologia, v. 139, p. 151-155, 1997.

PATRÍCIO, F.R.A., KIMATI, H., TESSARIOLI NETO, J., PETENATTI, A.; BARROS, B.C. Efeito da solarização, associada à aplicação de Trichoderma spp. ou fungicidas, sobre o controle de Pythium aphanidermatum e de Rhizoctonia solani AG-4. Summa Phytopathologica, v. 33, n. 2, p. 142-146, 2007.

PBMC. Sumário Executivo do Volume 1 - Base Científica das Mudanças Climáticas. Contribuição do Grupo de Trabalho 1 para o $1^{\circ}$ Relatório de Avaliação Nacional do Painel Brasileiro de Mudanças Climáticas. Volume Especial para a Rio+20. Rio de Janeiro, Brasil, 34 p, 2012.

PERCY, K.E.; AWMACK, C.S.; LINDROTH, R.L.; KUBISKE M.E.; KOPPER B.J.; ISEBRANDS J.G.; PREGITZER K. S.; HENDREY G.R.; DICKSON R.E.; ZAK D.R.; OKSANEN E.; SOBER J.; HARRINGTON R.; KARNOSKY D. F. Altered performance of forest pests under atmospheres enriched by $\mathrm{CO}_{2}$ and $\mathrm{O}_{3}$. Nature, v. 421, n. 6919, p. 190191, 2003.

RESENDE, M.L.; OLIVEIRA, J.A.; GUIMARÃES, R.M.; PINHO, R.G.V.; VIEIRA, A. R. Inoculações de sementes de milho utilizando o Trichoderma harzianum como promotor de crescimento. Ciência e Agrotecnologia, v. 28, n. 4, p. 793-798, 2004.

SILVA, F. F.; CASTRO, E. M.; MOREIRA, S. I.; FERREIRA, T. C.; LIMA, A.E.; ALVES, E. Emergência e análise ultraestrutural de plântulas de soja inoculadas com Sclerotinia sclerotiorum sob efeito da aplicação de Trichoderma harzianum. Summa Phytopathologica, v. 43, n. 1, p. 41-45, 2017.

VALADARES-INGLIS, M.C.; MARTINS, I.; DA SILVA, J.P.; MELLO, S.C.M.; Seleção in vitro de linhagens de Trichoderma para controle da podridão-branca do alho e da cebola. Brasília: Embrapa, 2018. 334 p.

License information: This is an open-access article distributed under the terms of the Creative Commons Attribution License (type CC-BY), which permits unrestricted use, distribution and reproduction in any medium, provided the original article is properly cited. 